\title{
Guaranteeing Robustness of Structural Condition Monitoring to Environmental Variability
}

\author{
Kendra Van Buren ${ }^{1}$ \\ Jack Reilly ${ }^{2}$ \\ Kyle $\mathrm{Neal}^{3}$ \\ Los Alamos National Laboratory \\ Los Alamos, New Mexico, U.S.A. \\ Princeton University \\ Princeton, New Jersey, U.S.A. \\ Vanderbilt University \\ Harry Edwards ${ }^{4}$ \\ Atomic Weapons Establishment \\ François Hemez ${ }^{5}$ \\ Reading, United Kingdom \\ Los Alamos National Laboratory \\ Los Alamos, New Mexico, U.S.A.
}

\begin{abstract}
Advances in sensor deployment and computational modeling have allowed significant strides to be recently made in the field of Structural Health Monitoring (SHM). One widely used SHM strategy is to perform a vibration analysis where a model of the structure's pristine (undamaged) condition is compared with vibration response data collected from the physical structure. Discrepancies between model predictions and monitoring data can be interpreted as structural damage. Unfortunately, multiple sources of uncertainty must also be considered in the analysis, including environmental variability, unknown model functional forms, and unknown values of model parameters. Not accounting for these sources of uncertainty can lead to falsepositives or false-negatives in the structural condition assessment. To manage the uncertainty, we propose a robust SHM methodology that combines three technologies. A time series algorithm is trained using "baseline" data to predict the vibration response, compare predictions to actual measurements collected on a potentially damaged structure, and calculate a userdefined damage indicator. The second technology handles the uncertainty present in the problem. An analysis of robustness is performed to propagate this uncertainty through the time series algorithm and obtain the corresponding bounds of variation of the damage indicator. The uncertainty description and robustness analysis are both inspired by the theory of info-gap decision-making. Lastly, an appropriate "size" of the uncertainty space is determined through physical experiments performed in laboratory conditions. Our hypothesis is that examining how the uncertainty space changes throughout time might lead to superior diagnostics of structural damage as compared to only monitoring the damage indicator. This methodology is applied to a portal frame structure to assess if the strategy holds promise for robust SHM. (Publication approved for unlimited, public release on October-28-2015, LA-UR-15-28442, unclassified.)
\end{abstract}

Keywords: Structural health monitoring, time series modeling, uncertainty quantification. 


\section{Introduction}

In the discipline of Structural Health Monitoring (SHM), the condition of a structure is often assessed using a combination of physical measurements and predictions from mathematical or numerical models $[1,2]$. The paradigm is to infer the structural condition from changes in the vibration response [3-6]. A structure inevitably degrades as it ages, through cycles of thermal loading, structural loading, and other conditions that affect structural health. As a result of this structural degradation, at some point, the structure may no longer be able to meet its performance requirements. This work contributes to the vast body of literature that develops diagnostics to detect such changes before they become safety or mission critical $[7,8]$.

To detect the onset of changes that could adversely affect structural integrity, the state-of-thepractice is to attach sensors to the structure and measure its vibration response from external excitation. Commonly, accelerometers and strain gauges are used to measure the structure's response [9, 10]. For in-service structures, ambient excitations such as cars on a bridge, wind over a building, or ground vibrations are typically used to elicit the vibration response [11, 12]. In contrast, it is common to perform deliberate and controlled excitation such as a modal hammer impact strike or modal shaker for laboratory tests. Diagnostics of structural health can be sought by analyzing changes to the Frequency Response Function (FRF) data, which is appropriate for dynamics that remain mostly linear and stationary $[13,14]$. Another approach is to train a time series model on the pristine condition of the structure, and then use the model to predict the structure's response and assess if its condition remains unchanged [15-17]. Shifts in natural frequencies observed from the FRF data, or deviations from predictions of time series models, could indicate structural damage $[14,18]$. It is emphasized that this process is only effective if measurements of the structure in its current state can be compared to measurements obtained from the pristine structure, which form a known "baseline." In the absence of baseline test data, mathematical modeling can be substituted to create theoretical "data" of the pristine state [19].

In this study, a time series model from the family of Auto Regressive (AR) representations [20] is used to analyze the acceleration response data (measurements) from an aluminum frame structure tested in controlled laboratory settings. The AR model is trained using multiple sets of vibration responses collected while the structure is in a "pristine," or undamaged, condition. The hypothesis is that the occurrence of damage manifests itself as a significant difference between what is measured on the (now damaged) structure and what the trained AR model predicts. The difference in structural condition, from undamaged to damaged, is diagnosed by large prediction residuals. A damage indicator is proposed that derives from statistics of the prediction residuals.

While the aforementioned strategy is well accepted within the SHM community, one challenge, which remains for the most part unresolved, is the quantification of uncertainty. A change in vibration signature does not necessarily indicate that the structure is damaged. It might, instead, be due to environmental variability, such as a change in temperature or input excitation [21]. To avoid the possibility of generating false-positives or false-negatives, it is highly desirable to "separate" the effects of environmental variability from those of structural damage.

Another unavoidable source of uncertainty is lack-of-knowledge associated to the model used to predict the vibration response. For example, when using the AR representation, the choice of model order has been shown to affect the assessment of structural health [20]. Further, when training a model of arbitrary mathematical form, its parameters might be non-unique whereby multiple sets of parameter values are able to replicate the training data with comparable fidelity. It is highly desirable to "separate" the effects of model-form uncertainty from those of structural damage. In this work, the effects of environmental uncertainty are quantified by replicating the vibration tests when the input excitation signal is varied within reasonable bounds. Likewise, the effects of modeling uncertainty are quantified by analyzing, not a single best-fitted time series 
model, but a family of models that includes all representations that fit the measurements with a similar level of accuracy. The effects of these two main sources of uncertainty (environmental variability and model-form uncertainty) are quantified such that the structural damage detection can be rendered robust to their unavoidable occurrence [22].

The description of environmental variability and modeling lack-of-knowledge is inspired by the theory of information-gap (or info-gap) for decision-making [23]. The magnitude, or "size," of the uncertainty, which the decision ("is the structural state pristine or damaged?") must be robust to, is controlled by a horizon-of-uncertainty parameter denoted by $\alpha$. A larger value of $\alpha$ increases the uncertainty considered in the analysis and, therefore, allows for greater potential deviation between reality and the numerical model used to predict the structural state. References [24] and [25] provide examples of defining the horizon-of-uncertainty $\alpha$, and performing an analysis of robustness, for applications to groundwater flow and transient dynamics, respectively.

This research aims to assess structural conditions in a manner that is immune (robust) to uncertainty. To do so, two concepts are combined. Firstly, the maximum horizon-of-uncertainty, $\alpha$, required to capture the environmental variability and modeling lack-of-knowledge for the pristine state is determined. This is a conventional analysis of robustness such as proposed, for instance, in References [22-23]. Secondly, damage is diagnosed by observing whether $\alpha$ increases, which would indicate "growth" of the uncertainty space, as the (potentially damaged) structure progressively deviates from the pristine state. The novelty is to assess how $\alpha$ changes over time, after having set a "baseline" that represents the environmental variability. While embedding an info-gap model of uncertainty within damage detection has been previously achieved (see, for example, an application to neural networks in Reference [26]), monitoring the growth of the uncertainty space is an aspect unique to this work. Another enhancement is the ability to account for the correlation structure of our uncertainty variables (see Section 4), which matters greatly to avoid over-estimating the prediction of damage. Preliminary results indicate the success of this strategy. Damage can be reliably diagnosed from observing the extent to which the horizon-of-uncertainty increases, while offering immunity (robustness) to the environmental variability and modeling lack-of-knowledge.

\section{Experimental Procedure}

The test structure considered is a two-story portal frame made of aluminum bars and steel brackets with bolted connections as shown in Figure 1-a (left). The structure is clamped to the support table with four C-clamps. A modal shaker is bolted to one of the columns at $40.7 \mathrm{~cm}$ above the base plate. The structure is excited with a chirp sine wave whose frequency varies from 1,000 Hertz down to 2 Hertz, and to which uniform white noise is added. It generates input excitation signals whose amplitudes and frequency contents vary from test to test, which mimics realistic testing conditions. It is verified that the applied chirp signal excites the lower resonant frequencies of the structure, which helps to capture a more descriptive response envelope.

White noise is added to the input shaker signal to vary the excitation for each test performed. A noise-to-signal ratio of $10 \%$ uniform noise is used. It produces output measurements that vary slightly from test to test, which simulates the aggregate effect of environmental variability (manufacturing variability, assembling variability, slight changes in material properties, etc.) on the structural response. This procedure introduces one of the sources of environmental variability considered in the problem; the other sources are described later.

To capture the response, four accelerometers are attached to the structure, as indicated by the red box in Figure 1-a (left). A detail is provided in Figure 1-b (right), where the green box shows the location of one of the accelerometers. Two sensors are located such that they can measure acceleration in the same direction as that of shaker input, while the other two are oriented to 
capture the out-of-plane response. Only the data collected at the location of the green box in Figure 1-b (right) are used for the analysis reported herein.

Vibration tests are performed over several days to accumulate sixty sets of pristine response data. The controlled laboratory settings in which the experiments are conducted significantly limit the day-to-day ambient conditions that have the potential to add variability to the measurements. Temperature fluctuations, for example, are limited to $\pm 2.0{ }^{\circ} \mathrm{C}$, which does not change the elasticity modulus of aluminum by more than $\pm 0.01 \%$. Using high-intensity lighting spots to heat the frame structure was considered. It was quickly dismissed due to the several hours that would have been required to produce a significant enough level of thermal expansion in the frame structure. The main source of variability in the vibration response of the pristine condition was operator-to-operator variability, that is, the fact that different analysts performed the vibration tests. Our contention is that assessing damage should be made as immune as possible, or robust, to the presence of these sources of environmental variability.

Two independent sets of vibration tests are executed to characterize the pristine condition. The first set is performed before the structure was modified to introduce different damage scenarios. The second set is performed after testing four of the damage cases. Assessing the statistical consistency of the response provides confidence that the "baseline" (pristine) state of the structure has not significantly shifted due to the various tests performed.

Damage is introduced in the structure in a number of different ways, as summarized in Table 1. Firstly, mass is added in the form of one and then two C-clamps attached to the middle plate. These cases are labeled II and III in Table 1. A single C-clamp weights approximately $1 \%$ of the total mass of the portal frame. The mass loading that results is believed to induce a linear change in the vibration response of the structure. Secondly, the bolt located in the top-right connection is loosened (Case-IV). This case potentially generates nonlinear vibrations from the impact between the vertical column, horizontal plate, and attachment bracket of the portal frame. An advantage of the time series modeling pursued in this work is that it can be used to detect linear and nonlinear changes to the response alike. Thirdly, Case- $V$ replaces the bottomright vertical column with a thinner (skinnier) and subsequently lighter column and Case-VI removes three of the four bolts that connect the vertical column to the horizontal base plate. While its response remains linear, Case- $V$ defines a significant structural change. Case-VI introduces a potential non-linearity in the vibration response because of the higher likelihood for column-to-base-plate impact.

Table 1. Definition of structural states (pristine, variability, damage) considered.

\begin{tabular}{|c|l|c|c|c|}
\hline Case & \multicolumn{1}{|c|}{ Description } & Variability? & Damage? & Replicates \\
\hline $0^{(I)}$ & First set of pristine (before cases I to V) & Yes & No & 60 \\
\hline $0^{(I I)}$ & Second set of pristine (after cases I to V) & Yes & No & 20 \\
\hline I & Table kicked during vibration testing & Yes (Extreme) & No & 20 \\
\hline II & One C-clamp added to middle plate & No & Yes & 20 \\
\hline III & Two C-clamps added to middle plate & No & Yes & 20 \\
\hline IV & Loosening of top-right connection bolt & No & Yes & 20 \\
\hline V & Thinner, lighter bottom-right column & No & Yes & 20 \\
\hline VI & Removal of three base-plate bolts & No & Yes & 20 \\
\hline
\end{tabular}

Table 1 also includes Case-I that keeps the frame structure in its pristine condition, identical to cases $0^{(I)}$ and $0^{(I I)}$, while a member of the team "kicks" the support table during the execution of 
vibration tests. This unorthodox procedure is meant to define an extreme case of environmental variability where the excitation provided to the portal frame is not even stationary. The table lists the numbers of replicates performed for each case. It can be observed that this experimental campaign results in a significant number of vibration tests $(60+7 \times 20=200$ tests $)$.

The third and fourth columns of Table 1 attempt to categorize the perturbations introduced to the frame structure as either "variability" or "damage." The mass loading cases (II and III) are meant to represent, for example, a heavy piece of equipment that would be installed inside a building. Loosening a connecting bolt defines a clear damage scenario. It is unclear how Case-I, and its extreme form of exciting the structure by kicking the support table, should be categorized. Our main objective is to demonstrate that the damage cases (II, III, IV, V, and VI) can be separated from the pristine cases $\left(0^{(I)}\right.$ and $\left.0^{(I I)}\right)$ in the presence of environmental variability represented by replicate testing of the pristine structure across multiple days. Another success would be to demonstrate that Case-I is not confused for structural damage.

\section{Diagnosis of Structural Damage in the Presence of Environmental Variability}

The procedure being implemented to diagnose the occurrence of structural damage from vibration measurements is to train a time series model using "baseline" data that represent the pristine structural state. Baseline model predictions are compared to actual measurements collected on a potentially damaged structure, and a damage indicator is calculated. This section summarizes the procedure and explains how the experimental variability is handled.

We start by considering three time series models for analysis of the raw acceleration signals: an Auto Regressive (AR) model, an Auto Regressive Moving Average (ARMA) model, and an Auto Regressive Exogenous (ARX) model [27]. Equation (1) shows the generic form of these models, which represent the measurements using a linear combination of input and output responses:

$$
\hat{y}_{k}=\sum_{1 \leq j \leq N} \beta_{j} \cdot y_{k-j}+\sum_{1 \leq j \leq M} \gamma_{j} \cdot f_{k-j}+e_{k}
$$

where $f_{k}$ denotes the input excitation (force applied by the shaker) at time sample $t_{k}, y_{k}$ denotes the output response (acceleration measured by the accelerometer), and $e_{k}$ is a statistical whitenoise term that accounts for model-fitting errors. The symbol $\hat{y}_{k}$ is the prediction of the model, which can be compared to the actual measurement, $y_{k}$, to define the prediction residual, $\varepsilon_{k}$ :

$$
\varepsilon_{k}=y_{k}-\hat{y}_{k}
$$

In equation (1), the optimal values of orders $(N ; M)$ of AR and MA summations are determined by repeating the training procedure, as discussed in the following. Once the AR and MA model orders are decided, the regression coefficients $\left(\beta_{j} ; \gamma_{j}\right)$ are fitted to the "baseline" data.

An AR model is restricted to the first summation, that is, $M=0$ in equation (1), which defines a linear combination of $N$ past observations $\left(y_{k-1} ; y_{k-2} ; \ldots y_{k-N}\right)$. An ARMA model is comprised of the AR term to which a contribution that represents an exogenous input is added. In the case of an unknown excitation signal, this additional term can be defined as the residual (2) obtained from AR predictions. These residuals $\varepsilon_{k}$ are then substituted to the input $f_{k}$ in equation (1).

An ARMA model is initially selected to model the vibration response of the frame structure. A study is undertaken to determine the optimal orders $(N ; M)$ of summations in equation (1). Both orders are varied to analyze all $(N ; M)$ combinations ranging from $1 \leq N \leq 25$ for the AR term and $1 \leq M \leq 30$ for the MA term. The mean absolute value of prediction residuals (2) is used as a metric for goodness-of-fit between actual measurements $\left(y_{k}\right)$ and predictions $\left(\hat{y}_{k}\right)$ of the model. 
Figure 2 indicates how the mean statistics of residual error vary as a function of orders $(N ; M)$. These results are obtained for one of the replicate tests of the pristine structure and it is verified that similar trends are observed with the other replicates. A lower residual mean value describes a model that predicts the measured acceleration response more accurately. From Figure 2, it is clear that, first, an AR order of $N=10$ suffices to "stabilize" the residual error and, second, the MA term does not seem to have a significant impact on the goodness-of-fit.

In light of the results illustrated in Figure 2, it is decided to conduct our investigation with an AR model. Figure 3 shows the Root Mean Square (RMS) value of AR prediction errors (2) for model orders that range in $1 \leq N \leq 100$. The figure indicates that a model order of $N=10$ is sufficient to reach an acceptable level of prediction accuracy while avoiding over-fitting the measurements. For completeness, it is mentioned that ARX models are investigated in a similar manner, with no improvement in accuracy over that of AR models. Results discussed in the remainder of the manuscript are obtained with AR models of order $N=10$.

After having selected the modeling approach (AR) and model order $(N=10)$, the ten unknown regression coefficients $\left(\beta_{1} ; \ldots \beta_{10}\right)$ of equation (1) are best-fitted to acceleration measurements for each replication of Case- $0^{(1)}$, see Table 1. The "baseline" AR model of the pristine condition is defined by averaging the regression coefficients across replicate tests:

$$
\left\langle\beta_{j}\right\rangle=\frac{1}{R} \cdot \sum_{1 \leq r \leq R} \beta_{j}^{(r)}
$$

where the superscript $(\bullet)^{(r)}$ identifies one of the replicate tests, that is, $1 \leq \mathrm{r} \leq R$, and brackets $<\bullet>$ indicate an averaged value. Figure 4 illustrates this procedure, which yields the averaged coefficients, $\left\langle\beta_{1}\right\rangle \ldots\left\langle\beta_{10}\right\rangle$, of the "baseline" AR model.

Once the "baseline" AR model is available to characterize the response of the pristine condition, it is analyzed to predict the structural state given a new set of experimental vibration data. The predictions are compared to actual measurements to calculate the residual errors (2). The mean value and standard deviation value of prediction residuals are estimated and used to define our Damage Indicator (DI) as a combination of the two statistics:

$$
D I=\left(\frac{\mu}{\mu_{0}}\right)+\left(\frac{\sigma}{\sigma_{0}}\right)
$$

where $\mu$ and $\sigma$ denote the mean and standard deviation values, respectively. In equation (4), the symbols $\mu_{0}$ and $\sigma_{0}$ are "reference" values used to scale the two contributions to $D I$. Scaling is needed because the mean statistics are observed to be several orders of magnitude smaller than the standard deviation values. Figure 5 illustrates the procedure to calculate $\mathrm{DI}$. It is noted that many strategies are conceivable to define the residual error for a damage indicator. One could, for example, directly compare measurements collected for the damaged state to those obtained for the pristine state. While the procedure implemented in this work has been shown to perform well for a wide range of applications [27-28], it is acknowledged that other definitions of the residual error have not been pursued and should be investigated in future research.

While the damage indicator defined in equation (4) perform satisfactorily for our application, it is acknowledged that more versatile metrics can be found in the literature. Reference [15] surveys well-established indicators of structural damage. Other examples are provided in References [29-30] for vibration-based assessments and wind turbine applications. Since the purpose of this study is to develop a robust SHM solution, as opposed to deriving a novel damage indicator, no attempt is made herein to search for a more powerful damage metric.

The sixty replicate tests defined for Case- $0^{(1)}$ are divided into training and validation sets. Fifty tests are used for training the "baseline" AR model, that is, $R=50$ in Figure 4 . The remaining 
ten tests are utilized to assess how the model performs to predict data that have not been used for training, which is the validation step. The "reference" statistics $\left(\mu_{0} ; \sigma_{0}\right)$ of equation (4) are estimated from the residual errors of these ten validation tests. Since replicates of the Case- $0^{(l)}$ pristine condition are slightly different because of environmental variability (see Section 2), this procedure is deemed appropriate to capture its effect on predictions of the "baseline" AR model.

The "baseline" model is defined from multiple replicates of the pristine state. Thus, generating the $\mathrm{DI}$ as shown in Figure 5 with a new time series measurement collected from the structure in a pristine condition should result in a low magnitude of residual error. The corresponding value of the DI should be close to 2, which would represent similar statistics as those obtained when analyzing the measurements used for training. Processing a time series measurement that represents a different structural state, on the other hand, should generate prediction residuals whose magnitude exceeds that produced by environmental variability alone. The value of the DI metric should increase. This is how we expect to separate the effect of structural damage from that of environmental variability, irrespective of the vibration dynamics (linear or nonlinear).

After training the "baseline" AR model to represent the pristine condition, the process of Figure 5 is applied to each one of the twenty replicate tests of Case-I, Case-II, Case-III, and Case-IV, see Table 1. Within any one of these configurations, the excitation signal applied to the structure differs slightly from test to test due, as mentioned earlier, to the addition of random white noise. It means that the vibration response of the portal frame varies slightly as well for each test. This variability propagates to the prediction residuals, their statistical moments, and the DI metrics.

Figure 6 represents the distributions of replicate DI values obtained for the four perturbed states as ranges that extend from the minimum to maximum values. Each color (red, magenta, green, and black) corresponds to one of cases I to IV. The blue interval indicates the range of DI values that result from replicates of the Case- $0^{(l)}$ pristine condition. Black crosses denote the average values of the test replicates.

For each configuration tested, the ranges of DI values capture the effects of the environmental variability. The question is whether the structural perturbations of Cases I to IV produce shifts of DI that can be separated from the aforementioned ranges. This is clearly observed for Case-II (magenta interval) and Case-III (green interval), which perturb the nominal configuration of the frame structure through mass loading. The conclusion is similar for Case-IV (black range) where one of the column-to-plate connecting bolts is loosened. The ranges obtained for these three configurations are completely outside the DI bounds obtained for the pristine structure. These cases, therefore, can be confidently classified as damaged. It is interesting to notice that cases II and III register such large DI shifts, even though the mass added is modest (1\% to $3 \%$ ).

The red interval, representing the table "quake" of Case-I where the support table is kicked during vibration testing, cannot be unambiguously identified as either damaged or undamaged. This is because the interval partially overlaps with the DI bounds of the pristine structure, as indicated in Figure 6. This perturbation represents a case of extreme variability but not damage. Because the training data do not encompass such a significant variability, the DI bounds of the pristine state (blue interval) do not fully overlap with DI values obtained for Case-I.

The table "quake" of Case-I stands out as a condition that our procedure cannot classify well. The preliminary results obtained nevertheless demonstrate the strong potential that this method offers to classify with accuracy the structural condition despite environmental variability.

\section{Rendering the Damage Assessment Robust to Modeling Lack-of-knowledge}

In this section, we discuss how to render the assessment of structural condition robust to the inevitable modeling lack-of-knowledge. This modeling uncertainty, here, is represented by 
the unknown regression coefficients, $\beta_{k}$, of the AR model. Even though it is not discussed here, other sources of modeling uncertainty could easily be included in the proposed methodology. The AR model order, for example, could be considered to be a modeling uncertainty as well as the functional form of the time series representation. Other models could include ARMA, support vector machine, Gaussian process, etc.

The objective of the proposed methodology is to guarantee that the assessment of structural condition (pristine, damaged?) is as robust as possible to the fact that parameters of the time series model are not known precisely. We would not want to find ourselves in a situation where, for example, the assessment of structural condition changes from damaged to undamaged, or vice-versa, simply because the values of model parameters shift slightly due to uncontrolled factors. Establishing robustness provides confidence that the decision reached "the structure is damaged") is correct despite the fact that model parameters vary from their nominal values.

To establish the robustness of condition monitoring, each regression coefficient of the "baseline" AR model is varied between a lower bound, $\beta_{k}^{\text {Lower }}$, and an upper bound, $\beta_{k}^{\text {Upper }}$, as:

$$
\left\langle\beta_{\mathrm{k}}\right\rangle+\alpha \cdot\left(\beta_{k}^{\text {Lower }}-\left\langle\beta_{\mathrm{k}}\right\rangle\right) \leq \beta_{k} \leq\left\langle\beta_{\mathrm{k}}\right\rangle+\alpha \cdot\left(\beta_{k}^{\text {Upper }}-\left\langle\beta_{\mathrm{k}}\right\rangle\right)
$$

where $\alpha$ denotes the dimensionless, "horizon-of-uncertainty" parameter greater or equal to zero. The lower and upper bounds are obtained from the population of model regression coefficients $\left(\beta_{k}{ }^{(1)} ; \beta_{k}{ }^{(2)} ; \ldots \beta_{k}{ }^{(R)}\right)$ calculated in Figure 4 during the training step of the pristine condition.

Equation (5) varies each regression coefficient, $\beta_{k}$, between lower and upper bounds depending on the value of parameter $\alpha$. With $\alpha=0$, there is no variation and the regression coefficient defaults back to its "baseline" value, that is, $\beta_{k}=\left\langle\beta_{k}\right\rangle$. With $\alpha=1$, the regression coefficient can take any value between within the interval $\beta_{k}^{\text {Lower }} \leq \beta_{k} \leq \beta_{k}^{\text {Upper }}$. It is noted that $\alpha$ is unbounded and can take any positive value, including $\alpha \geq 1$. In fact, it is precisely by observing that the horizon-of-uncertainty increases beyond $\alpha=1$ that damage is diagnosed in Section 4.

The procedure starts by setting the horizon-of-uncertainty parameter $\alpha$, then, repeating multiple times the assessment of structural condition of Figure 5 by varying the values of regression coefficients within the lower and upper bounds of equation (5). Each calculation of the DI metric is based on predictions of an AR model with coefficients $\left(\beta_{1} ; \ldots \beta_{10}\right)$ that deviate from the meanvalue coefficients $\left.\left(\left\langle\beta_{1}\right\rangle ; \ldots<\beta_{10}\right\rangle\right)$ of the "baseline" AR model. The magnitude of the deviation is controlled by $\alpha$. The parameter $\alpha$, therefore, defines the modeling uncertainty embodied, here, by unknown regression coefficients. The goal of robustness is to quantify how much uncertainty can be tolerated before the assessed structural condition changes from pristine to damaged.

When performing the robustness analysis, however, the ten model parameters cannot be varied independently from each other. This is because they are statistically correlated. Figure 7 depicts the correlation structure for pairs of regression coefficients $\left(\beta_{p} ; \beta_{q}\right)$ for $p \neq q$. The figure indicates a strong correlation for all pairs of model parameters except combinations $\left(\beta_{7} ; \beta_{8}\right)$ and $\left(\beta_{8} ; \beta_{9}\right)$.

Figure 8 is a notional illustration of what happens when the correlation structure is ignored as the regression coefficients $\left(\beta_{1} ; \ldots \beta_{10}\right)$ are generated. The green-dot points remain "true" to the nominal correlation suggested by the ellipsoid shape (blue dashed line). While sampling the model parameters, we wish to avoid generating red-cross points that fall outside the ellipsoid. Independently varying each model parameter according to equation (5) would generate samples that do not accurately map the modeling uncertainty space of the "baseline" AR representation.

To maintain the correlation between regression coefficients of Figure 7, a Principal Component Analysis (PCA) is implemented to transform the coefficients to a generalized coordinate space according to the decomposition: 


$$
\boldsymbol{\beta}-\langle\boldsymbol{\beta}\rangle=\mathbf{U} \cdot \boldsymbol{\Sigma} \cdot \mathbf{V}^{\mathrm{T}}
$$

where the symbol $\beta$ is the $N$-by- $R$ matrix of regression coefficients and $\langle\beta\rangle$ is a matrix of same size that contains the mean values $\left.\left(\left\langle\beta_{1}\right\rangle ; \ldots<\beta_{10}\right\rangle\right)$. Matrix $\boldsymbol{\beta}$ collects $N$ regression coefficients of the AR model for each one of the $R$ replicate tests. The model order is $N=10$ in our analysis and the number of replicates is $R=20$ (damage) or 60 (pristine). The triplet of matrices (U; $\boldsymbol{\Sigma} ; \mathbf{V})$ in the right-hand side of equation (6), which feature sizes $N$-by- $N$ (for $\mathbf{U}$ ), $N$-by- $N$ (for $\boldsymbol{\Sigma}$ ), and $N$ by- $R$ (for $\mathbf{V}$ ), identifies the PCA decomposition [31].

After calculating the triplet of matrices $(\mathbf{U} ; \boldsymbol{\Sigma} ; \mathbf{V})$ of the decomposition (6), it is used "in reverse" to generate correlated samples of regression coefficients, as illustrated notionally in Figure 9. The figure suggests that a vector of uncorrelated coordinates $\mathbf{v}^{(r)}=\left(v_{1}^{(r)} ; \ldots v_{10}{ }^{(r)}\right)$ sampled in the hypercube $([-\alpha ;+\alpha])^{10}$ produces a vector of correlated regression coefficients $\boldsymbol{\beta}^{(\mathrm{r})}=\left(\beta_{1}{ }^{(r)} ; \ldots \beta_{10}{ }^{(r)}\right)$ defined in the 10-dimensional "ellipsoid" whose axes are aligned in the direction of orthogonal vectors $\left(\mathbf{U}_{1} ; \ldots \mathbf{U}_{10}\right)$, and whose magnitudes are given by the singular values stored in matrix $\boldsymbol{\Sigma}$. This procedure defines an info-gap model of uncertainty [23], which can be formally written as:

$$
\mathrm{U}(\langle\boldsymbol{\beta}\rangle ; \alpha)=\left\{\boldsymbol{\beta}^{(\mathbf{r})} \text { such that } \boldsymbol{\beta}^{(\mathbf{r})}=\langle\boldsymbol{\beta}\rangle+\mathbf{U} \cdot \boldsymbol{\Sigma} \cdot \mathbf{v}^{(\mathbf{r})^{\mathrm{T}}} \text { and }\left\|\mathbf{v}^{(\mathbf{r})}\right\| \leq \alpha\right\}
$$

where $\mathbf{v}^{(\mathbf{r})}$ denotes a realization of right singular vector and $\left\|\mathbf{v}^{(\mathbf{r})}\right\|=\left(\mathbf{v}^{(\mathbf{r}) \top} \mathbf{v}^{(\mathbf{r})}\right)^{1 / 2}$ is the Euclidean norm of a vector defined from its inner product.

Because the coordinates $\left(v_{1}^{(r)} ; \ldots v_{10}^{(r)}\right)$ are uncorrelated, Latin Hypercube Sampling (LHS) is implemented to sample their values according to a uniform distribution [32]. The LHS method offers an efficient trade-off between randomness and spreading the samples within $([-\alpha ;+\alpha])^{10}$. Equation (6) is used to transform the uniform samples back to correlated space. This procedure is implemented in Matlab ${ }^{\circledR}$ and only takes a fraction of second to complete when implemented on a single-core desktop processor.

A Monte Carlo simulation is analyzed using 10,000 sets of regression coefficients $\left(\beta_{1}{ }^{(r)} ; \ldots \beta_{10}{ }^{(r)}\right)$ obtained using the combination of PCA transform and LHS method previously described. The $\beta$ values are consistent with the lower and upper bounds of equation (5) where $\alpha=1$. A value of the $\mathrm{DI}$ is obtained for each Monte Carlo sample, following the flowchart of Figure 5 , and with the only difference that samples $\left(\beta_{1}^{(r)} ; \ldots \beta_{10}{ }^{(r)}\right)$ are substituted to the mean values $\left(<\beta_{1}>; \ldots<\beta_{10}>\right)$.

Figure 10 depicts the 10,000 samples obtained for the pair of model parameters $\left(\beta_{1} ; \beta_{2}\right)$, which features the strongest correlation of all pairs $\left(\beta_{p} ; \beta_{q}\right), p \neq q$. Red crosses represent the training data of the Case- $0^{(1)}$ pristine condition and blue dots are the 10,000 new samples created from equation (5) at the level of modeling uncertainty of $\alpha=1$. The figure indicates that the overall correlation is preserved by our sampling algorithm, as suggested by a coefficient of correlation between $\beta_{1}$ and $\beta_{2}$ equal to $-97.6 \%$ for the training points and $-97.9 \%$ for the new samples.

Figure 10 also suggests that the level of modeling uncertainty used, $\alpha=1$, generates variations of model parameters $\left(\beta_{1}{ }^{(r)} ; \ldots \beta_{10}{ }^{(r)}\right)$ that exceed those observed when replicating the vibration tests for the Case- $0^{(1)}$ pristine configuration. The perturbations of model parameters generated in this manner account for variability not directly observed during vibration testing and that could manifest itself for that specific structural state at another time. In addition, these perturbations are appropriate to explore the modeling lack-of-knowledge, that is, the fact that adopting a different time series modeling approach or changing the model order could result in a largerthan-currently-observed variation of model parameters. The robustness question is: "does this variability of AR model parameters change the assessment of structural condition?"

The above question is answered by running a Monte Carlo simulation of 10,000 LHS-generated AR models through each case defined in Table 1, including the training, support table "quake," 
and damage cases. Figure 11 displays the ranges of DI values obtained from the analysis of each pristine and damage configuration (20 sets of data each) run through these 10,000 AR models. The difference with Section 3 is that Figure 6 only assesses fluctuations of DI values caused by experimental variability when the parameters of the AR model do not change, that is, $\beta_{k}=\left\langle\beta_{k}\right\rangle$, as shown in the flowchart of Figure 5 . The ranges of DI values reported here combine the effect of experimental variability and the effect of model-form uncertainty, that is, the fact that AR model coefficients are varied between the lower and upper bounds as defined in equation (5) for $\alpha=1$.

It can be observed in Figure 11, firstly, that the DI ranges for the two independent tests of the pristine condition, Case- $0^{(\mathrm{I})}$ (orange interval) and Case- $0^{(\mathrm{II})}$ (yellow interval), are remarkably consistent. This gives confidence that our procedure is not adversely affected by small changes to the "baseline" state that result from modifying the frame structure multiple times and testing it over-and-over. A second finding is that Case-I (purple interval), which represents the table "quake" where the support table is kicked during testing, is consistent with the pristine states. This result is welcome since this configuration, while it represents an extreme form of variability, is not, strictly speaking, damaged. The consistency of DI bounds for the three sets, Case- $0^{(1)}$ (orange), Case- $0^{(\mathrm{II})}$ (yellow), and Case-I (purple), provides a "calibration" of the horizon-ofuncertainty parameter, $\alpha$, needed to capture all pristine states of the frame structure. This analysis is predicated on a rather large number of trials (20 replicates by 10,000 samples $=$ 200,000 trials), which supports the rigorousness of our exploration of the uncertainty space.

DI bounds predicted for the pristine states overlap, for the most part, when the modeling lack-ofknowledge represented by equation (5) is explored up to the horizon-of-uncertainty of $\alpha=1$. The figure also indicates that the DI bounds of pristine configurations are approximately distinct from those of the damaged states (Case-II, magenta interval, Case-III, light blue interval, and CaseIV, green interval). Generating DI bounds for damaged states that partially overlap with those of pristine states would mean that false positives (assessing that the condition is damaged when it is not) or false negatives (assessing that the condition is pristine when it is not) are possible, which we wish to avoid. Said differently, $\alpha=1$ is the largest size of the uncertainty space that separates the DI bounds of pristine and damaged states, which is written mathematically as:

$$
\propto^{*}=\max \left\{\propto \geq 0 \text { such that }\left[D I_{\text {Min }}^{(P)} ; D I_{\text {Max }}^{(P)}\right] \cap\left[D I_{\text {Min }}^{(D)} ; D I_{\text {Max }}^{(D)}\right]=\emptyset \text { for all } \boldsymbol{\beta} \in U(\langle\boldsymbol{\beta}\rangle ; \propto)\right\}
$$

where $\left[D I_{\text {Min }}{ }^{(P)} ; D I_{M a x}{ }^{(P)}\right]$ denotes the range of $D I$ values for pristine states and $\left[D I_{M i n}{ }^{(D)} ; D I_{M a x}{ }^{(D)}\right]$ is the range for damage states. The criterion of non-overlapping intervals and equation (7) define the performance requirement and uncertainty space, respectively, which are the essential attributes of a robustness analysis [23]. Equation (8) is the robust solution because $U\left(\langle\beta\rangle ; \alpha^{*}\right)$ is the largest-possible uncertainty space for which the criterion of non-overlapping intervals is met.

For our application, the structural condition is robustly diagnosed, up to $\alpha=1$, irrespective of the uncertainty of the vibration testing protocol (nominal testing vs. support table "shake") and environmental variability (test-to-test replication). This success should not hide the caveat that it can be challenging to achieve robustness when predictions are extrapolated away from conditions represented in the training data. "How far" a model can be extrapolated to settings away from training data is an area of open research, not just in SHM but also in computational sciences and engineering. Clearly, the success of the methodology proposed here for robust damage detection hinges on the ability to properly characterize the experimental variability in the training data.

As $\alpha$ increases, so does the uncertainty space, $\mathrm{U}(\langle\beta\rangle ; \alpha)$. It yields model parameters $\left(\beta_{1} ; \ldots \beta_{10}\right)$ that increasingly deviate from "baseline" values $\left.\left(\left\langle\beta_{1}\right\rangle ; \ldots<\beta_{10}\right\rangle\right)$ of the pristine structural state. 
The result is that model predictions, as well as statistics of prediction residuals in equation (4), implicitly depend on $\alpha$. The appendix analyzes this dependence by deriving a closed-form upper bound of damage indicator as a function of $\alpha$. For the specific definition of DI used here, this functional dependence is a combination of half, linear, and quadratic powers of $\alpha$.

One could conceive, therefore, increasing the horizon-of-uncertainty, $\alpha$, until the DI bounds of pristine conditions start overlapping with those of damaged states. Total overlap would mean increasing the upper bound from $D I_{\operatorname{Max}}{ }^{(P)}=3.5$, which is the maximum observed for a pristine state (Case-I), to $D I_{\operatorname{Max}}{ }^{(D)}=9.0$, which is the maximum observed for a damaged state (Case-II). From equations $(A-16)$ and $(A-18)$ of the appendix, increasing the DI value can be achieved by changing the horizon-of-uncertainty from $\alpha^{(P)}$ to $\alpha^{(D)}$ such that $\left(\alpha^{(D)} / \alpha^{(P)}\right)^{\eta}=D I_{M a x}{ }^{(D)} / D I_{M a x}{ }^{(P)}$. With $\alpha^{(P)}=1.0$ in Figure 11 and exponents $\eta=(1 / 2 ; 1 ; 2)$, one obtains $\alpha^{(D)} \approx(6.6 ; 2.6 ; 1.6)$. The corresponding spaces $U\left(\langle\beta\rangle ; \alpha^{(D)}\right)$ are a significant departure from the space $U\left(\langle\beta\rangle ; \alpha^{(P)}\right)$ inferred from "calibration" of the pristine states, which reinforces the conclusion that Case-II, Case-III, and Case-IV are damaged. These results illustrate the potential offered by our strategy for robust SHM, whereby the growth of the uncertainty space, $U(\langle\beta\rangle ; \alpha)$, is quantified as opposed to simply monitoring the value of a damage metric.

In most real-world applications, it will not be possible to derive the functional dependence of a damage indicator with $\alpha$. Numerical optimization can be substituted to closed-form derivations to search for the range of damage indicators over uncertainty spaces $U(\langle\beta\rangle ; \alpha)$ for increasing $\alpha$ values. How challenging it is to solve these optimization problems can depend on, first, the dimensionality of the problem, that is, how many variables introduce uncertainty in the analysis, and, second, the computational resource required to evaluate the damage detection algorithm. Another practical challenge might be to obtain the solution in a reasonable time for a complex application. These issues, however, are not fundamental bottlenecks as they can be mitigated with access to a more powerful computational resource and parallel processing. References [33-34] provide examples of how to implement numerical optimization to search for the minimum and maximum values of a performance metric over increasingly larger uncertainty spaces.

Lastly, Figure 11 shows that the procedure does not successfully "isolate" the configurations of Case-V (orange interval, thinner column) and Case-VI (dark blue interval, removal of three base bolts) from the pristine condition. These two damage cases represent slightly less severe alterations of the vibration dynamics than those of Case-II/Case-III (mass loading) and Case-IV (loosened bolt). We hypothesize that it is the reason why they remain undetected. In addition, these scenarios introduce structural perturbations at the bottom-left side of the portal frame, while the measurements analyzed are collected from a single sensor located at the top-right. Even though this conjecture has not been verified, sensor data from the other accelerometers, or the addition of more sensors, could have possibly provided a better classification.

\section{Conclusion}

A commonly encountered strategy to deploy Structural Health Monitoring (SHM) solutions is to develop a mathematical or numerical model that represents a given condition, such as the pristine (undamaged) state, and to compare predictions to actual data collected on the structure. Discrepancies between the two sets are interpreted as a change in condition and, potentially, structural damage. This strategy generally leaves unaddressed the difficult question of how to handle the sources of uncertainty that can include environmental variability, unknown functional forms of the mathematical models, and non-unique model parameter values.

To meet this challenge, we propose a framework where the assessment of structural condition is made robust to the aforementioned sources of uncertainty. Instead of monitoring the value of a damage index, we suggest to monitor the "growth" of the uncertainty space that describes the 
environmental variability and modeling lack-of-knowledge. An increasing level of uncertainty indicates that the structural condition deviates more-and-more from the nominal, "baseline" state and, therefore, is suspected to be evolving towards damage.

This novel concept is illustrated by testing a portal frame structure in different configurations that include pristine, mass-loaded, and damaged states. We demonstrate that the appropriate "size" of the uncertainty space, which describes both environmental variability and unknown values of model parameters, can be learned from analyzing replicate measurements of the pristine state. The damage detection procedure is based on developing an auto-regressive time series model, comparing its predictions to measurements, and summarizing the results in the form of a scalarvalued damage indicator. We demonstrate that, when it is embedded within the analysis of robustness, this algorithm is capable of separating the various states of the frame structure. A component essential to the success of this technology is the ability to explore model parameters while preserving their correlation structure. This is achieved by using a correlation-preserving sampling scheme based on the principal component decomposition of the correlation.

Our hypothesis, which is that examining how the uncertainty space changes in time might lead to superior diagnostics of structural damage as compared to only monitoring a damage index, is confirmed by these preliminary results. While this methodology holds promise for robust SHM, future research efforts should consider incorporating more of the statistical information that, while it is generated in our analysis, was not fully exploited. Other potential improvements would be to, first, generalize the procedure to multiple-input, multiple-output time series modeling and, second, develop more sophisticated indicators of damage. Another indicator would clearly offer different robustness properties. Future efforts could consider searching for damage indicators that, instead of attempting to be more sensitive to damage, would offer enhanced robustness.

\section{Acknowledgments}

The first author acknowledges support from the Advanced Scientific Computing program at Los Alamos National Laboratory (LANL). The second, third, and fourth authors acknowledge the support of the 2015 Dynamics Summer School at LANL. The fifth author is grateful for continued support received from the Advanced Certification Campaign at LANL. The Los Alamos National Security, L.L.C., operates LANL under contract DE-AC52-06NA25396 on behalf of the National Nuclear Security Administration of the U.S. Department of Energy.

\section{Appendix: Derivation of Upper Bounds of Damage Indicator Values}

This appendix derives an upper bound of the damage indicator to assess how it changes when increasingly more experimental variability and modeling lack-of-knowledge are accounted for in the analysis. From equation (4), the damage indicator is defined as:

$$
D I=\left(\frac{\mu}{\mu_{0}}\right)+\left(\frac{\sigma}{\sigma_{0}}\right)
$$

where $\mu$ and $\sigma$ are the mean and standard deviation statistics of prediction residuals written as:

$$
\varepsilon_{k}^{(r)}=y_{k}^{(\text {Test, } r)}-\left(\beta_{1}^{(r)} \cdot y_{k-1}^{(\text {Test, }, r)}+\cdots+\beta_{N}^{(r)} \cdot y_{k-N}^{(\text {Test, } r)}\right)=y_{k}^{(\text {Test }, r)}-\Psi_{\mathbf{k}}^{(\mathbf{r})^{\mathrm{T}}} \cdot \boldsymbol{\beta}^{(\mathbf{r})}
$$

In equation (A-2), $\boldsymbol{\beta}^{(r)}$ denotes the $N$-by-1 vector of AR model coefficients, $\boldsymbol{\beta}^{(r)}=\left(\beta_{1}{ }^{(r)} ; \ldots \beta_{N}{ }^{(r)}\right)$, and $\boldsymbol{\Psi}_{\mathbf{k}}{ }^{(\mathbf{r})}$ is the $N$-by-1 vector of past observations, $\boldsymbol{\Psi}_{\mathbf{k}}{ }^{(\mathbf{r})}=\left(y_{k-1}{ }^{(T e s t, r)} ; \ldots y_{k-N}{ }^{(T e s t, r)}\right)$. A superscript $(\bullet)^{(r)}$ is used to emphasize that quantities $\boldsymbol{\beta}^{(r)}$ and $\boldsymbol{\Psi}_{\mathbf{k}}{ }^{(r)}$ are defined for the $r^{\text {th }}$ replicate experiment $(1 \leq r \leq R)$. With this compact notation, the statistics $(\mu ; \sigma)$ of prediction residuals are:

$$
\mu=\left\langle\varepsilon_{k}^{(r)}\right\rangle=\left\langle y_{k}^{(\text {Test }, r)}\right\rangle-\left\langle\boldsymbol{\Psi}_{\mathbf{k}}^{(\mathbf{r})^{\mathrm{T}}} \cdot \boldsymbol{\beta}^{(\mathbf{r})}\right\rangle
$$


and:

$$
\sigma=\sqrt{\left\langle\left(y_{k}^{(\text {Test }, r)}-\boldsymbol{\Psi}_{\mathbf{k}}^{(\mathbf{r})^{\mathrm{T}}} \cdot \boldsymbol{\beta}^{(\mathbf{r})}-\mu\right)^{2}\right\rangle}
$$

where $<\bullet>$ represents the operator that averages over replicate experiments:

$$
\langle *\rangle=\frac{1}{R} \cdot \sum_{1 \leq \mathrm{r} \leq R} *
$$

Given these definitions, the upper bounds of statistics $(\mu ; \sigma)$ sought are denoted by:

$$
|\mu| \leq U_{\mu}(\alpha), \quad|\sigma| \leq U_{\sigma}(\alpha)
$$

To derive these bounds as a function of $\alpha$, the representation of uncertainty, which expresses experimental variability, must first be defined. Recall the decomposition of equation (6):

$$
\boldsymbol{\beta}=\langle\boldsymbol{\beta}\rangle+\mathbf{U} \cdot \boldsymbol{\Sigma} \cdot \mathbf{V}^{\mathrm{T}}
$$

where $\beta$ collects the AR model coefficients into a $N$-by- $R$ rectangular matrix for $N$ coefficients and $R$ replicate experiments ( $R=20$ or 60 and $N=10$ in our application). A single realization of model coefficients is obtained as:

$$
\boldsymbol{\beta}^{(\mathbf{r})}=\langle\boldsymbol{\beta}\rangle+\mathbf{U} \cdot \boldsymbol{\Sigma} \cdot \mathbf{v}^{(\mathbf{r})^{\mathrm{T}}}
$$

In equation $(\mathrm{A}-8)$, the symbol $\boldsymbol{\beta}^{(\mathrm{r})}=\left(\beta_{1}{ }^{(r)} ; \ldots \beta_{N}{ }^{(r)}\right)$ denotes a $N$-by-1 vector of model coefficients, $\mathbf{U}$ is the $N$-by- $N$ orthogonal matrix of left singular vectors (same as earlier), and $\boldsymbol{\Sigma}$ is the diagonal matrix of singular values (same as earlier). The 1 -by- $N$ quantity $\mathbf{v}^{(\mathbf{r})}$ represents the right singular vector of the $r^{\text {th }}$ replicate whose amplitude does not exceed the horizon-of-uncertainty, $\alpha$ :

$$
\left\|\mathbf{v}^{(\mathbf{r})}\right\|=\sqrt{\mathbf{v}^{(\mathbf{r}) \cdot \mathbf{v}^{(\mathbf{r})^{\mathrm{T}}}}} \leq \alpha
$$

The procedure to derive the upper bounds of DI values consists of propagating the uncertainty expressed in equation (A-9) to model coefficients $\boldsymbol{\beta}^{(\mathbf{r})}$, the prediction residuals $\boldsymbol{\varepsilon}_{k}{ }^{(r)}$, and finally their statistics $(\mu ; \sigma)$. A summary of these closed-form derivations is provided for the mean, $\mu$. For conciseness and since they follow a similar approach, derivations for the standard deviation are not given. The final result is included from which the dependence of the upper bound on the horizon-of-uncertainty, $\alpha$, can be observed.

By applying the triangular inequality, that is, ||$x|-| y|| \leq|x-y| \leq|x|+|y|$, it can be verified that the mean statistic of equation (A-3) is bounded by:

$$
|\mu| \leq\left|\left\langle y_{k}^{(\text {Test }, r)}\right\rangle\right|+\left|\left\langle\boldsymbol{\Psi}_{\mathbf{k}}^{(\mathbf{r})^{\mathrm{T}}} \cdot \boldsymbol{\beta}^{(\mathbf{r})}\right\rangle\right|
$$

which indicates that $\mu$ "grows" as the average of the inner products $\left\langle\boldsymbol{\Psi}_{\mathrm{k}}{ }^{(\mathbf{r}) \top} \boldsymbol{\beta}^{(\mathrm{r})}\right\rangle$ that depend on model coefficients, $\boldsymbol{\beta}^{(r)}$. The question becomes to bound this term given that $\boldsymbol{\beta}^{(\mathrm{r})}$ belongs to the uncertainty space $U(\langle\beta\rangle ; \alpha)$ of equation (7). The Cauchy-Schwarz inequality, which provides an upper bound of the inner product between two vectors as $\mathbf{x}^{\top} \mathbf{y} \leq\|\mathbf{x}\|\|\mathbf{y}\|$, yields:

$$
\boldsymbol{\Psi}_{\mathbf{k}}^{(\mathbf{r})^{\mathrm{T}}} \cdot \boldsymbol{\beta}^{(\mathbf{r})} \leq\left\|\boldsymbol{\Psi}_{\mathbf{k}}^{(\mathbf{r})}\right\| \cdot\left\|\boldsymbol{\beta}^{(\mathbf{r})}\right\|
$$

Substituting in equation (A-10) gives:

$$
|\mu| \leq\left|\left\langle y_{k}^{(T e s t, r)}\right\rangle\right|+\left\langle\left\|\boldsymbol{\Psi}_{\mathbf{k}}^{(\mathbf{r})}\right\| \cdot\left\|\boldsymbol{\beta}^{(\mathbf{r})}\right\|\right\rangle
$$


The terms $\left\langle y_{k}{ }^{(T e s t, r)}>\right.$ (average of the measured responses at time $t_{k}$ ) and $\left\|\boldsymbol{\Psi}_{\mathbf{k}}{ }^{(\mathbf{r})}\right\|$ (Euclidean norm of the vector of measured responses acquired from time $t_{k-N}$ to time $t_{k-1}$ ) are constant and not a function of $\alpha$. Therefore, only the Euclidean norm of the vector $\boldsymbol{\beta}^{(r)}$ of model coefficients introduces a dependence on the horizon-of-uncertainty, $\alpha$.

Using definition (A-8), the inner product of vector $\boldsymbol{\beta}^{(\mathrm{r})}$ is equal to:

$$
\left\|\boldsymbol{\beta}^{(\mathbf{r})}\right\|^{2}=\boldsymbol{\beta}^{(\mathbf{r})^{\mathrm{T}}} \cdot \boldsymbol{\beta}^{(\mathbf{r})}=\left(\langle\boldsymbol{\beta}\rangle^{\mathrm{T}} \cdot\langle\boldsymbol{\beta}\rangle\right)+2 \cdot\left(\langle\boldsymbol{\beta}\rangle^{\mathrm{T}} \cdot \mathbf{U} \cdot \boldsymbol{\Sigma} \cdot \mathbf{v}^{(\mathbf{r})^{\mathrm{T}}}\right)+\left(\mathbf{v}^{(\mathbf{r})} \cdot \boldsymbol{\Sigma}^{2} \cdot \mathbf{v}^{(\mathbf{r})^{\mathrm{T}}}\right)
$$

The first term in the right-hand side is the Euclidean norm of the average vector of model coefficients, which is constant. Upper bounds can be derived for the second and third terms by applying the Cauchy-Schwarz inequality. It can be verified that the second term is bounded by:

$$
\left(\langle\boldsymbol{\beta}\rangle^{\mathrm{T}} \cdot \mathbf{U} \cdot \boldsymbol{\Sigma} \cdot \mathbf{v}^{(\mathbf{r})^{\mathrm{T}}}\right) \leq\left\|\boldsymbol{\Sigma} \cdot \mathbf{U}^{\mathrm{T}} \cdot\langle\boldsymbol{\beta}\rangle\right\| \cdot\left\|\mathbf{v}^{(\mathbf{r})}\right\| \leq \alpha \cdot\left\|\boldsymbol{\Sigma} \cdot \mathbf{U}^{\mathrm{T}} \cdot\langle\boldsymbol{\beta}\rangle\right\|
$$

The third term in the right-hand side of equation $(A-13)$ is bounded by:

$$
\left(\mathbf{v}^{(\mathbf{r})} \cdot \boldsymbol{\Sigma}^{2} \cdot \mathbf{v}^{(\mathbf{r})^{\mathrm{T}}}\right)=\left\|\boldsymbol{\Sigma} \cdot \mathbf{v}^{(\mathbf{r})^{\mathrm{T}}}\right\| \leq\left\|\boldsymbol{\Sigma}_{\mathbf{D}}\right\|^{2} \cdot\left\|\mathbf{v}^{(\mathbf{r})}\right\|^{2} \leq \alpha^{2} \cdot\left\|\boldsymbol{\Sigma}_{\mathbf{D}}\right\|^{2}
$$

where $\boldsymbol{\Sigma}_{\mathrm{D}}$ denotes the vector of singular values of decomposition $(\mathrm{A}-7)$, that is, $\boldsymbol{\Sigma}_{\mathrm{D}}=\left(\sigma_{1} ; \ldots \sigma_{N}\right)$. Substituting in equation (A-12) provides the upper bound for the mean statistic, $\mu$ :

$$
|\mu| \leq\left|\left\langle y_{k}^{(\text {Test }, r)}\right\rangle\right|+\left\langle\left\|\boldsymbol{\Psi}_{\mathbf{k}}^{(\mathbf{r})}\right\|\right\rangle \cdot \sqrt{B(\alpha)}
$$

where the positive scalar $B(\alpha)$ carries the dependence on the horizon-of-uncertainty, $\alpha$ :

$$
B(\alpha)=\|\langle\boldsymbol{\beta}\rangle\|^{2}+2 \cdot \alpha \cdot\left\|\boldsymbol{\Sigma} \cdot \mathbf{U}^{\mathrm{T}} \cdot\langle\boldsymbol{\beta}\rangle\right\|+\alpha^{2} \cdot\left\|\boldsymbol{\Sigma}_{\mathbf{D}}\right\|^{2}
$$

The derivations (not shown) for the standard deviation of prediction residuals follow a similar approach of combining the triangular and Cauchy-Schwarz inequalities, and the final result is:

$$
\begin{gathered}
\sigma^{2} \leq\left|\mu^{2}+\left\langle y_{k}^{(\text {Test }, r)}\right\rangle+2 \cdot \mu \cdot\left\langle\left\|\boldsymbol{\Psi}_{\mathbf{k}}^{(\mathbf{r})}\right\|\right\rangle \cdot \sqrt{B(\alpha)}+\left\langle\left\|\boldsymbol{\Psi}_{\mathbf{k}}^{(\mathbf{r})}\right\|^{2}\right\rangle \cdot B(\alpha)\right| \\
+2 \cdot\left|\mu \cdot\left\langle y_{k}^{(\text {Test }, r)}\right\rangle+\left\langle y_{k}^{(\text {Test }, r)} \cdot\left\|\boldsymbol{\Psi}_{\mathbf{k}}^{(\mathbf{r})}\right\|\right\rangle \cdot \sqrt{B(\alpha)}\right|
\end{gathered}
$$

From these results, it is observed that DI values increase as a combination of half, linear, and quadratic powers of the horizon-of-uncertainty, $\alpha$. This theoretical result is used in Section 4 to extrapolate the value of $\alpha$ that would be required to encompass the pristine states of Case- $0^{(1)}$, Case- $0^{(I I)}$, and Case-I and the damaged scenarios of Case-II, Case-III, and Case-IV under a common uncertainty space $U(\langle\beta\rangle ; \alpha)$. (See the discussion of Figure 11.)

\section{References}

[1] S.W. Doebling, C.R. Farrar, M.B. Prime, D.W. Shevitz, Damage identification and health monitoring of structural and mechanical systems from changes in their vibration characteristics: a literature review, Technical Report LA-13070-MS, Los Alamos National Laboratory, Los Alamos, NM, May 1996.

[2] H. Sohn, C.R. Farrar, F.M. Hemez, D.D. Shunk, D.W. Stinemates, B.R. Nadler, A review of structural health monitoring literature: 1996-2001, Technical Report LA-13976-MS, Los Alamos National Laboratory, Los Alamos, NM, Mar. 2004.

[3] J. Maeck, B. Peeters, G. De Roeck, Damage identification on the Z24 bridge using vibration monitoring, Smart Materials and Structures 10 (2001) 512-517. 
[4] E. Keller, A. Ray, Real-time health monitoring of mechanical structures, Structural Health Monitoring: An International Journal 2 (2003) 191-203.

[5] P.C. Chang, A. Flatau, S.C. Liu, Review paper: health monitoring of civil infrastructure, Structural Health Monitoring: An International Journal 2 (2003) 257-267.

[6] H. Van der Auweraer, B. Peeters, International research projects on structural health monitoring: an overview, Structural Health Monitoring: An International Journal 2 (2003) 341-358.

[7] D.H. Sigurdardottir, B. Glisic, On-site validation of fiber-optic methods for structural health monitoring: Streicker Bridge, Journal of Civil Structural Health Monitoring 5 (2015) 529549.

[8] M.T. Yarnold, F.L. Moon, Temperature-based structural health monitoring baseline for long-span bridges, Engineering Structures 86 (2015) 157-167.

[9] G. Wang, K. Pran, G. Sagvolden, G.B. Havsgård, A.E. Jensen, G.A. Johnson, S.T. Vohra, Ship hull structure monitoring using fibre optic sensors, Smart Materials and Structures 10 (2001) 472-478.

[10] M.D. Todd, G.A. Johnson, S.T. Vohra, Deployment of a fiber bragg grating-based measurement system in a structural health monitoring application, Smart Materials and Structures 10 (2001) 534-539.

[11] R. Brincker, L. Zhang, P. Andersen, Modal identification of output-only systems using frequency domain decomposition, Smart Materials and Structures 10 (2001) 441-445.

[12] M. Basseville, L. Mevel, A. Vecchio, B. Peeters, H. Van der Auweraer, Output-only subspace-based damage detection: application to a reticular structure, Structural Health Monitoring: An International Journal 2 (2003) 161-168.

[13] E. Figueiredo, G. Park, C.R. Farrar, K. Worden, J. Figueiras, Machine learning algorithms for damage detection under operational and environmental variability, Structural Health Monitoring: An International Journal 10 (2010) 559-572.

[14] N.M.M. Maia, J.M.M. Silva, E.A.M. Almas, R.P.C. Sampaio, Damage detection in structures: from mode shape to frequency response function methods, Mechanical Systems and Signal Processing 17 (2003) 489-498.

[15] H. Sohn, C.R. Farrar, Damage diagnosis using time series analysis of vibration signals, Smart Materials and Structures 10 (2001) 446-451.

[16] J.B. Bodeux, J.C. Golinval, Application of ARMAV models to the identification and damage detection of mechanical and civil engineering structures, Smart Materials and Structures 10 (2001) 479-489.

[17] K.K. Nair, A.S. Kiremidjian, K.H. Law, Time series-based damage detection and localization algorithm with application to the ASCE benchmark structure, Journal of Sound and Vibration 291 (2006) 349-368.

[18] O.S. Salawu, Detection of structural damage through changes in frequency: a review, Engineering Structures 19 (1997) 718-723.

[19] E. Görl, M. Link, Identification of damage parameters of a full-scale steel structure damaged by seismic loading, Smart Materials and Structures 10 (2001) 459-465.

[20] M. Fugate, H. Sohn, C.R. Farrar, Vibration-based damage detection using statistical process control, Mechanical Systems and Signal Processing 15 (2001) 707-721. 
[21] B. Peeters, J. Maeck, G. De Roeck, Vibration-based damage detection in civil engineering: excitation sources and temperature effects, Smart Materials and Structures 10 (2001) 518-527.

[22] C.J. Stull, F.M. Hemez, C.R. Farrar, On assessing the robustness of structural health monitoring technologies, Structural Health Monitoring: An International Journal 11 (2012) 712-723.

[23] Y. Ben-Haim, Info-Gap Decision Theory: Decisions Under Severe Uncertainty, Academic Press Publisher, Oxford, $2^{\text {nd }}$ Edition, 2006.

[24] D. O'Malley, V.V. Vesselinov, Groundwater remediation using the information gap decision theory, Water Resources Research 50 (2014) 246-256.

[25] F.M. Hemez, Y. Ben-Haim, Info-gap robustness for the correlation of tests and simulations of a nonlinear transient, Mechanical Systems and Signal Processing 18 (2004) 1443-1467.

[26] S.G. Pierce, K. Worden, G. Manson, A novel information-gap technique to assess reliability of neural network-based damage detection, Journal of Sound and Vibration 293 (2006) 96-111.

[27] C.R. Farrar, K. Worden, Structural Health Monitoring: A Machine Learning Perspective, Wiley Publisher, New York, NY, 2012.

[28] H. Sohn, D.W. Allen, K. Worden, C.R. Farrar, Statistical damage classification using sequential probability ratio tests, Structural Health Monitoring: An International Journal 2 (2003) 57-74.

[29] Y.J. Yan, L. Cheng, Z.Y. Wu, L.H. Yam, Development in Vibration-based Structural Damage Detection Technique, Mechanical Systems and Signal Processing 21 (2007) 2198-2211.

[30] C.C. Ciang, J.R. Lee, H.J. Bang, Structural health monitoring for a wind turbine system: a review of damage detection methods, Measurement Science and Technology 19 (2008) $1-20$.

[31] G. Kerschen, P. De Boe, J.C. Golinval, K. Worden, Sensor validation using principal component analysis, Smart Materials and Structures 14 (2005) 36-42.

[32] M.D. McKay, R.J. Beckman, W.J. Conover, A comparison of three methods for selecting values of input variables in the analysis of output from a computer code, Technometrics 21 (1979) 239-245.

[33] K.L. Van Buren, F.M. Hemez, Robust Decision-making applied to the NASA multidisciplinary uncertainty quantification challenge problem, AIAA Journal of Aerospace Information Systems 12 (2015) 35-48.

[34] K.L. Van Buren, S. Atamturktur, F.M. Hemez, Model selection through robustness and fidelity criteria: modeling the dynamics of the CX-100 wind turbine blade, Mechanical Systems and Signal Processing 43 (2014) 246-259. 


\section{LIST OF FIGURES}

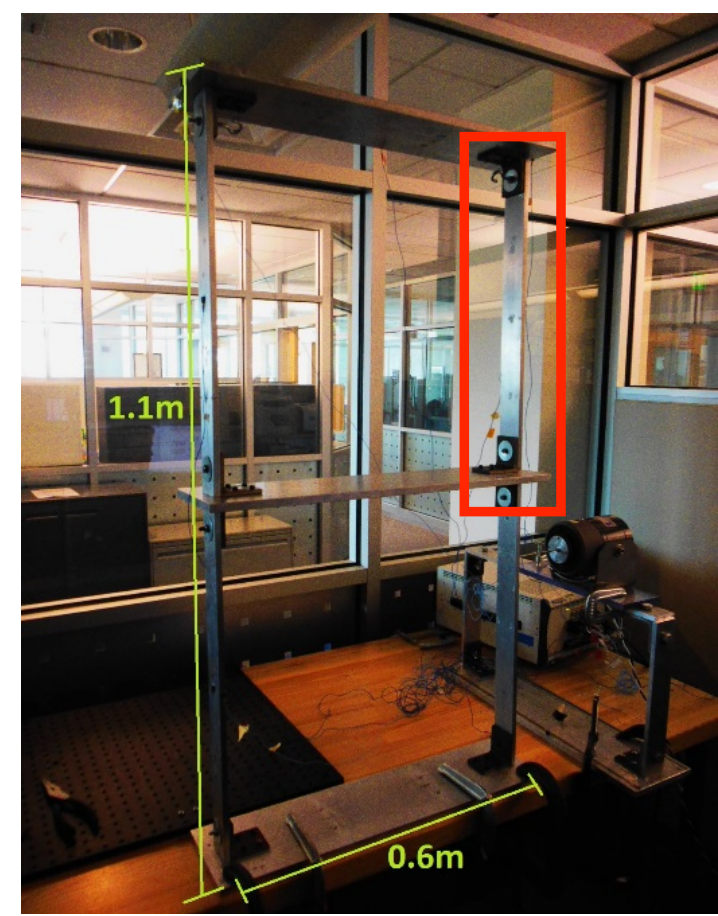

(a) Left: Test structure.

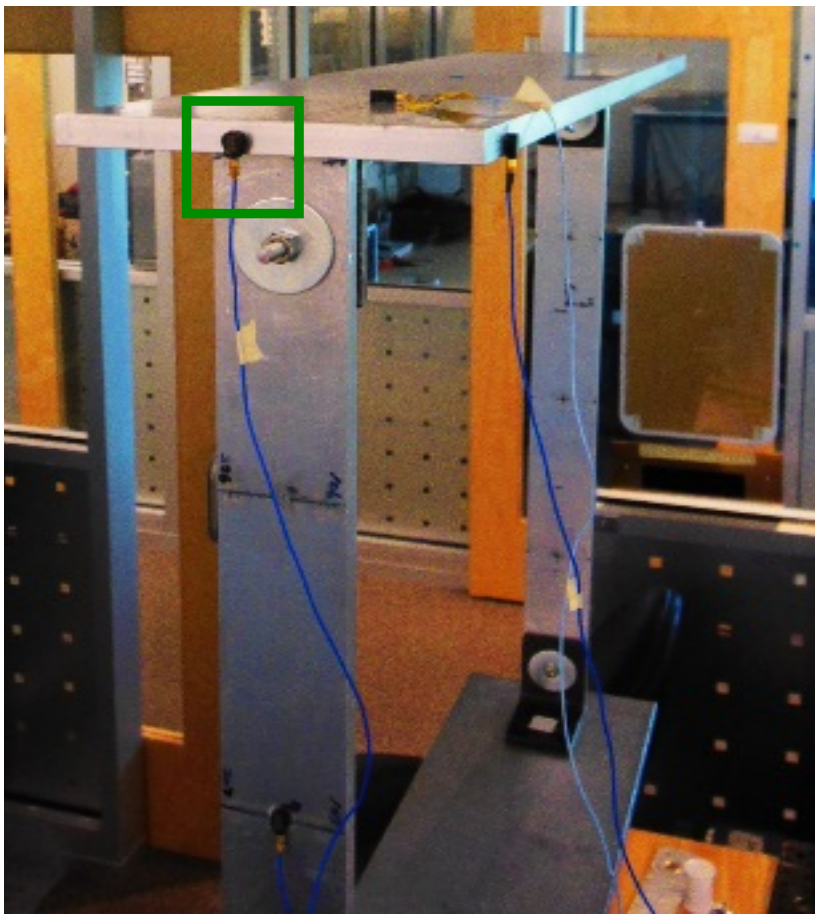

(b) Right: Sensor placement.

Figure 1. Portal frame structure instrumented to perform the vibration tests.

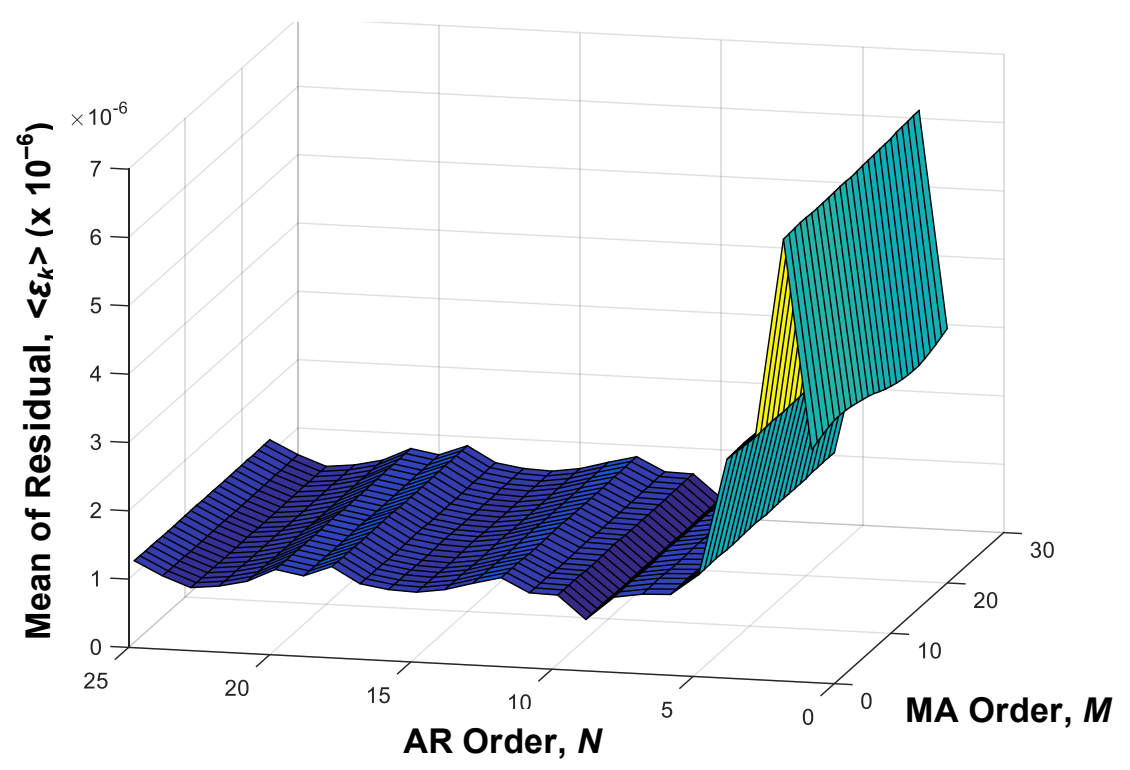

Figure 2. Mean values of ARMA residual errors for varying model orders $(N ; M)$. 


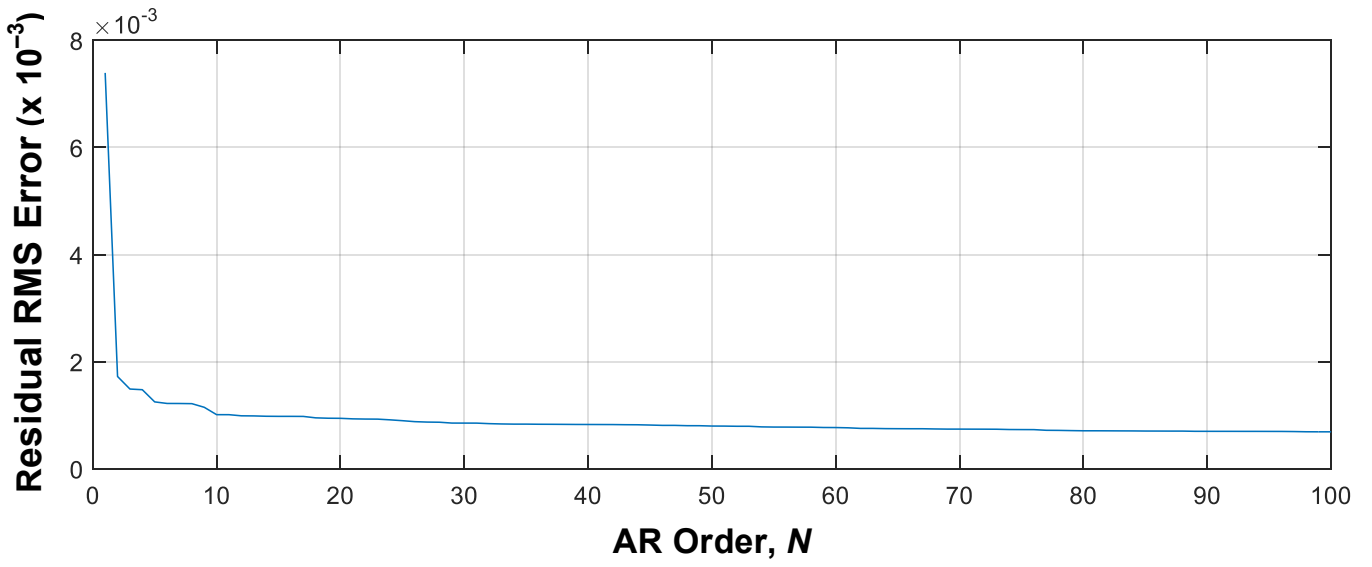

Figure 3. Mean values of AR residual errors for varying model orders, $1 \leq N \leq 100$. 


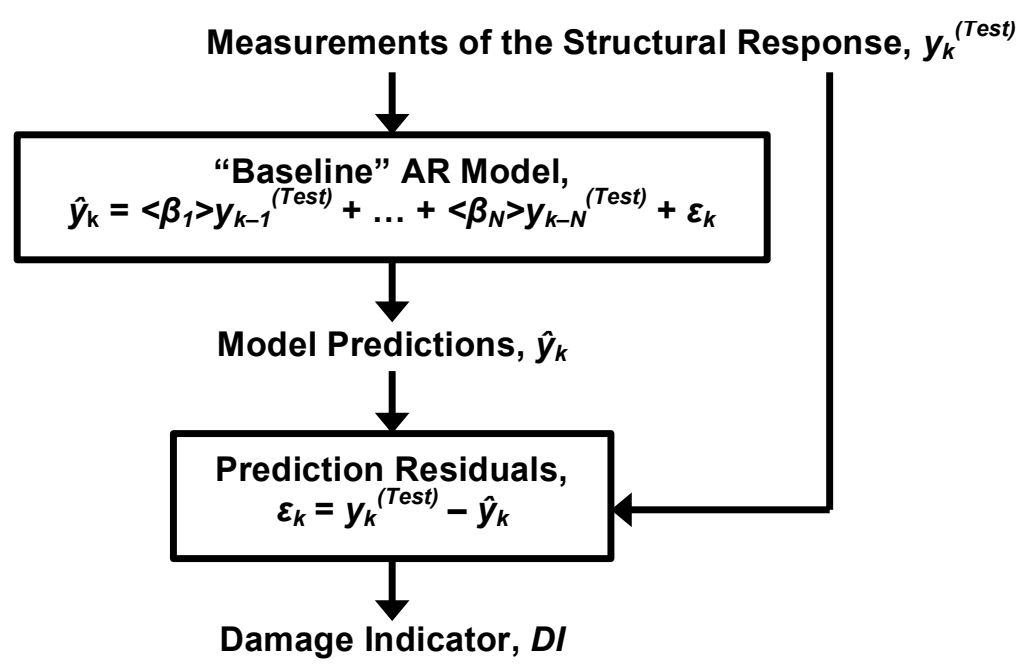

Figure 5. Damage indicator defined from the vibration test of a different structural state.

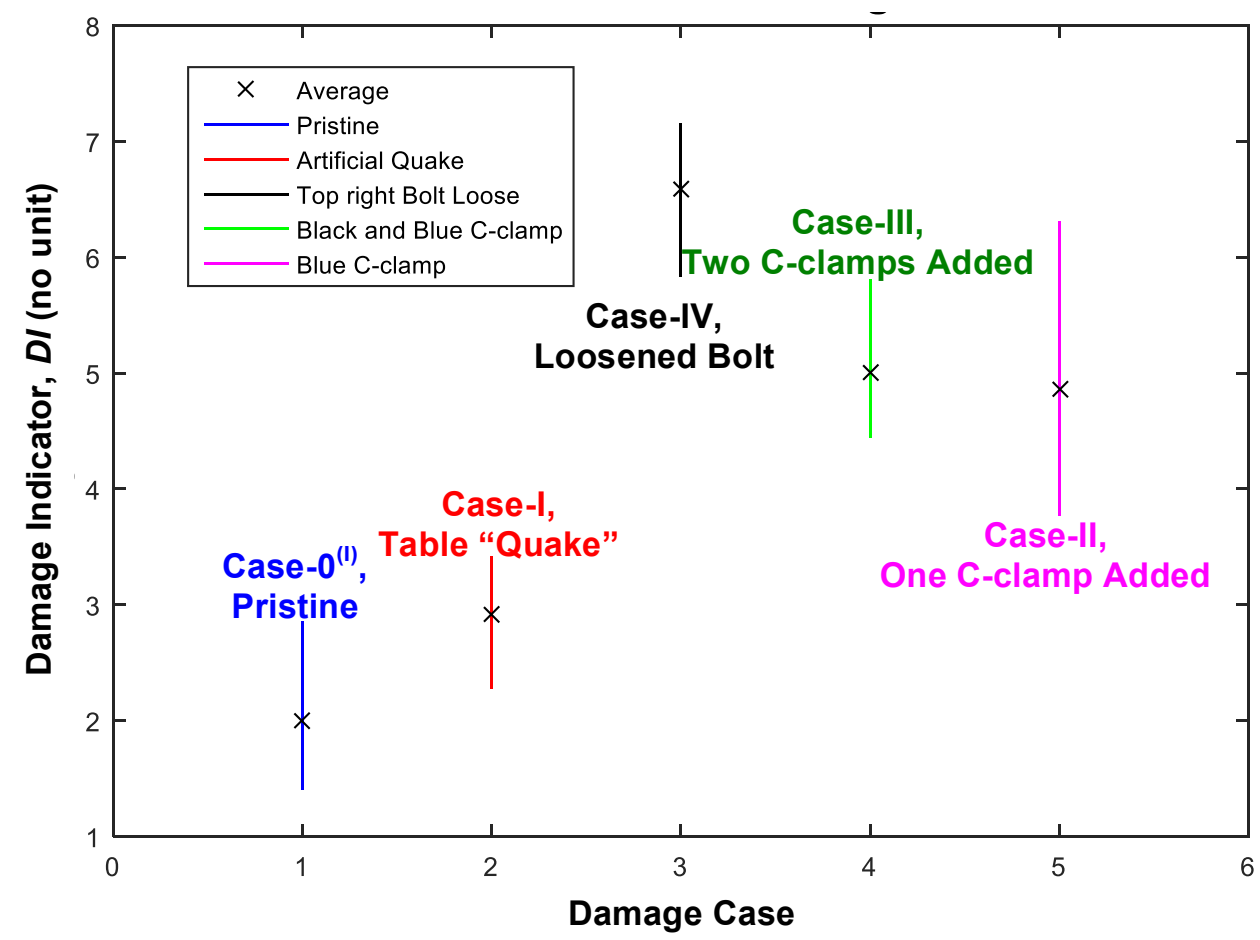

Figure 6. Damage indicators of Case- $0^{(\mathrm{I})}$ (pristine) and cases I to IV (perturbed). 

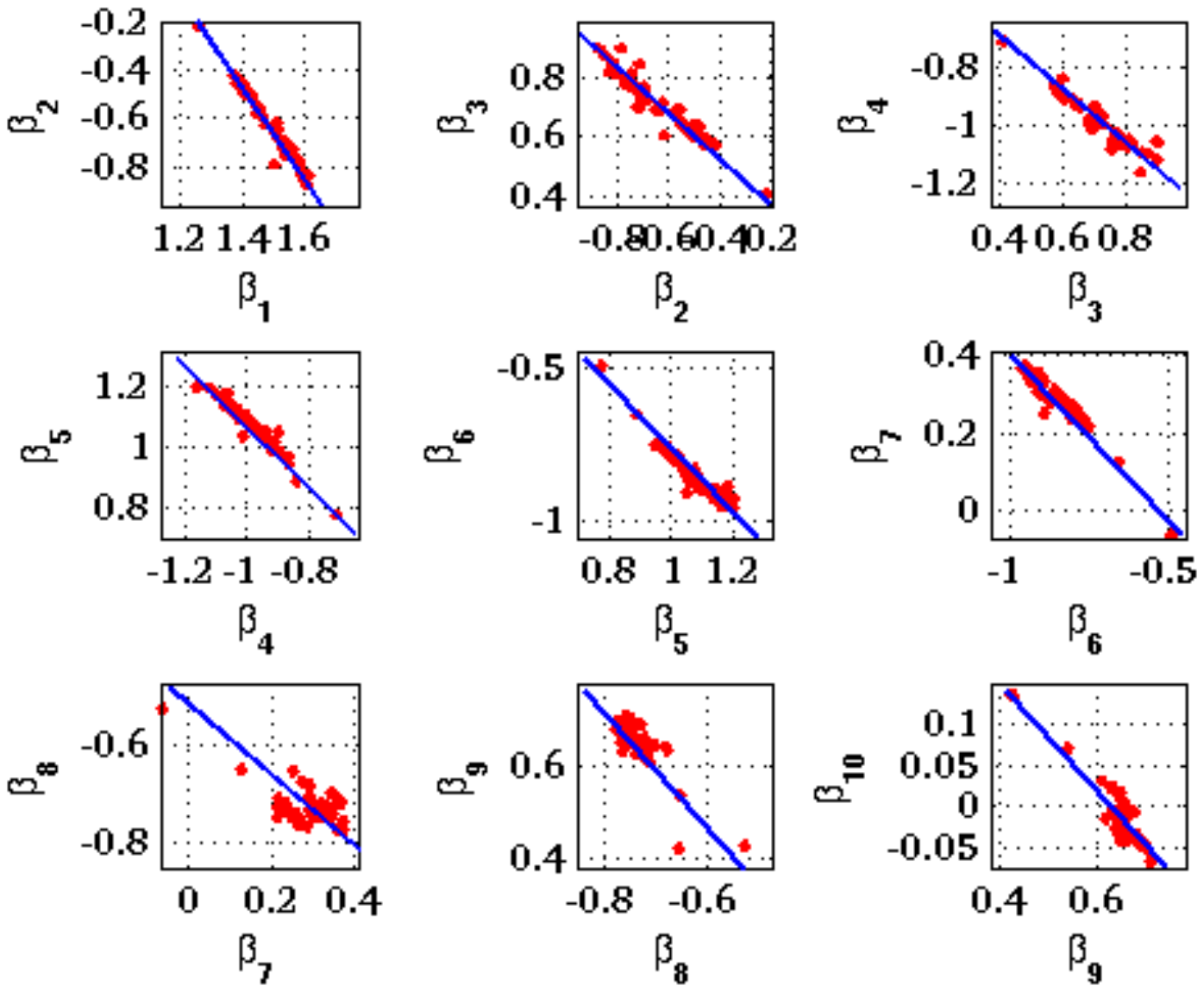

Figure 7. Pairs $\left(\beta_{p} ; \beta_{q}\right), p \neq q$, of AR regression coefficients obtained for Case-0 ${ }^{(l)}$.

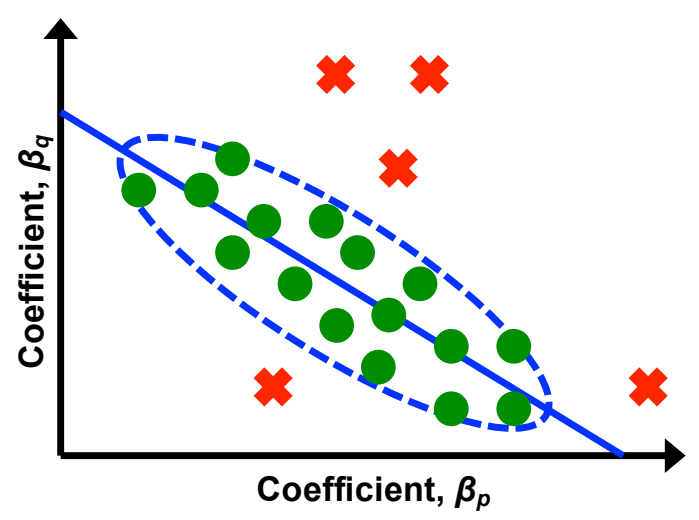

Figure 8. Coefficients sampled inside (green dot) or outside (red cross) the correlation. 


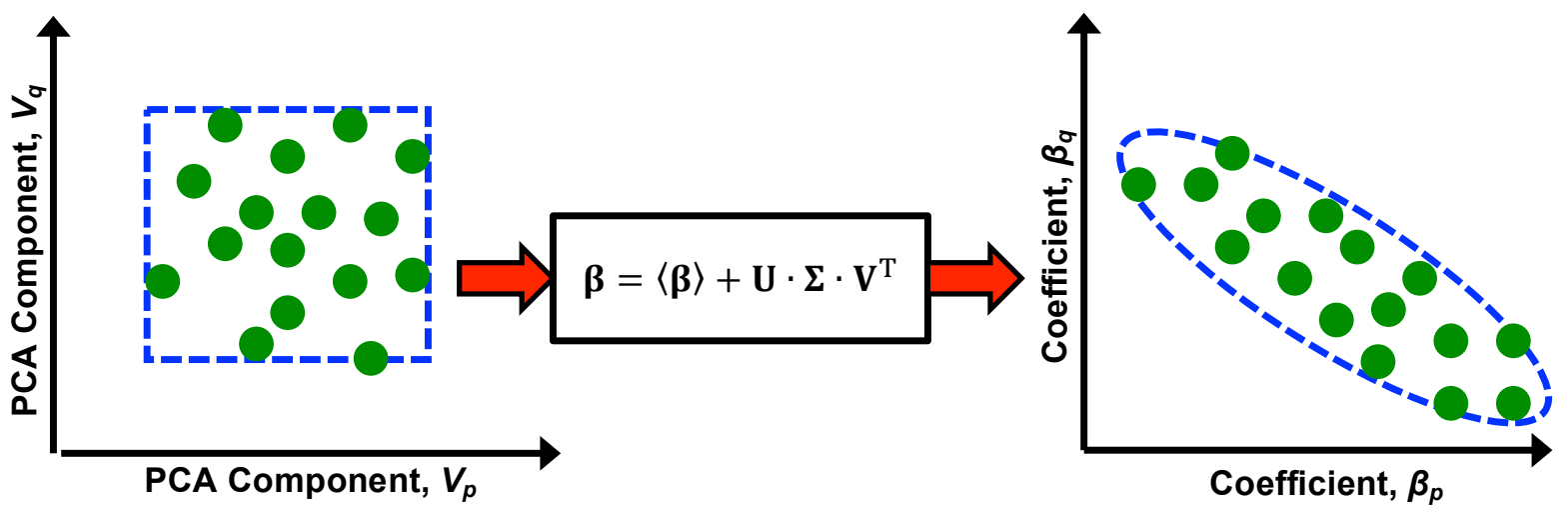

Figure 9. Transform implemented to sample the regression coefficients $\left(\beta_{1} ; \ldots \beta_{10}\right)$.

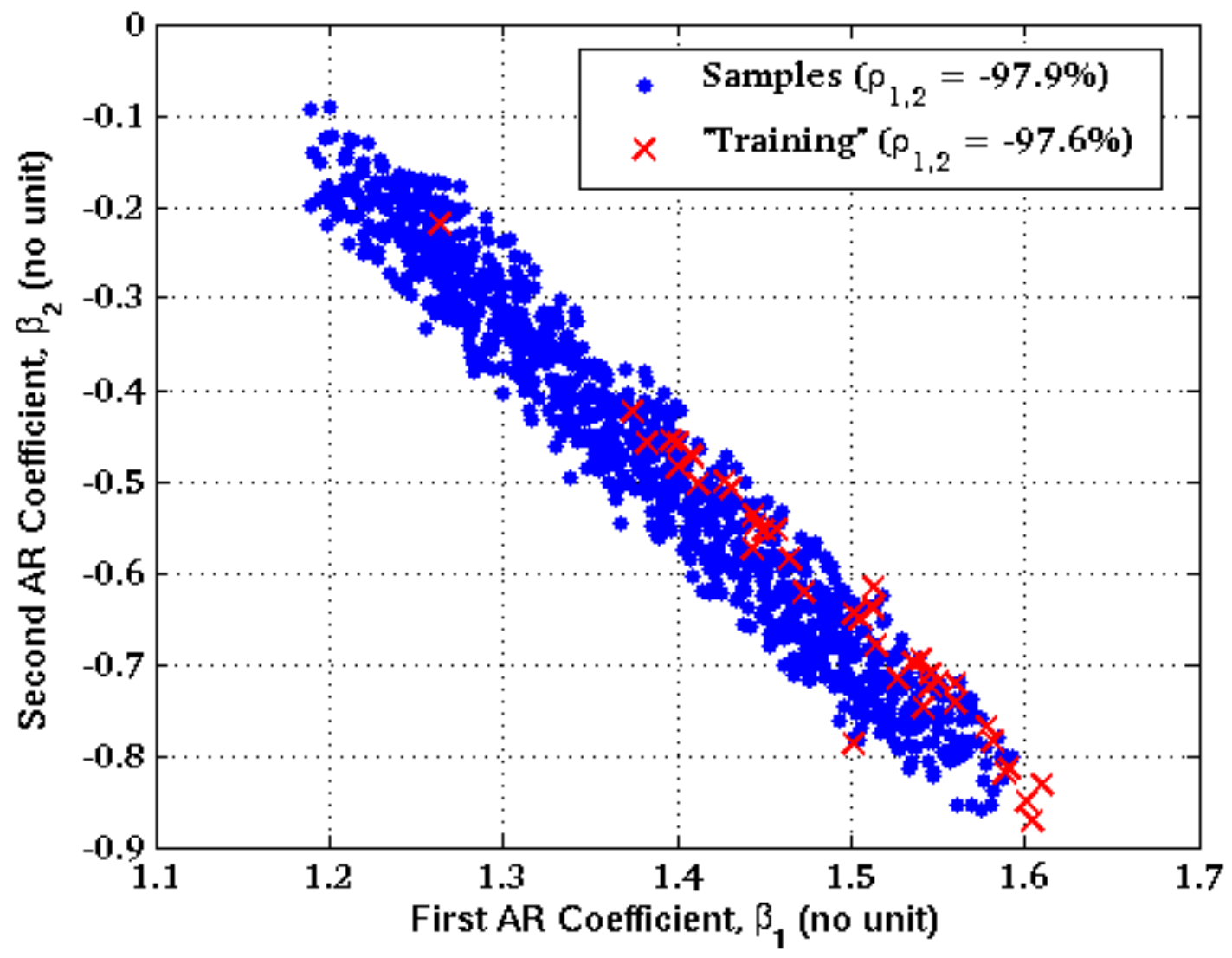

Figure 10. Nominal and new correlation structure for the pair $\left(\beta_{1} ; \beta_{2}\right)$ of AR coefficients. 


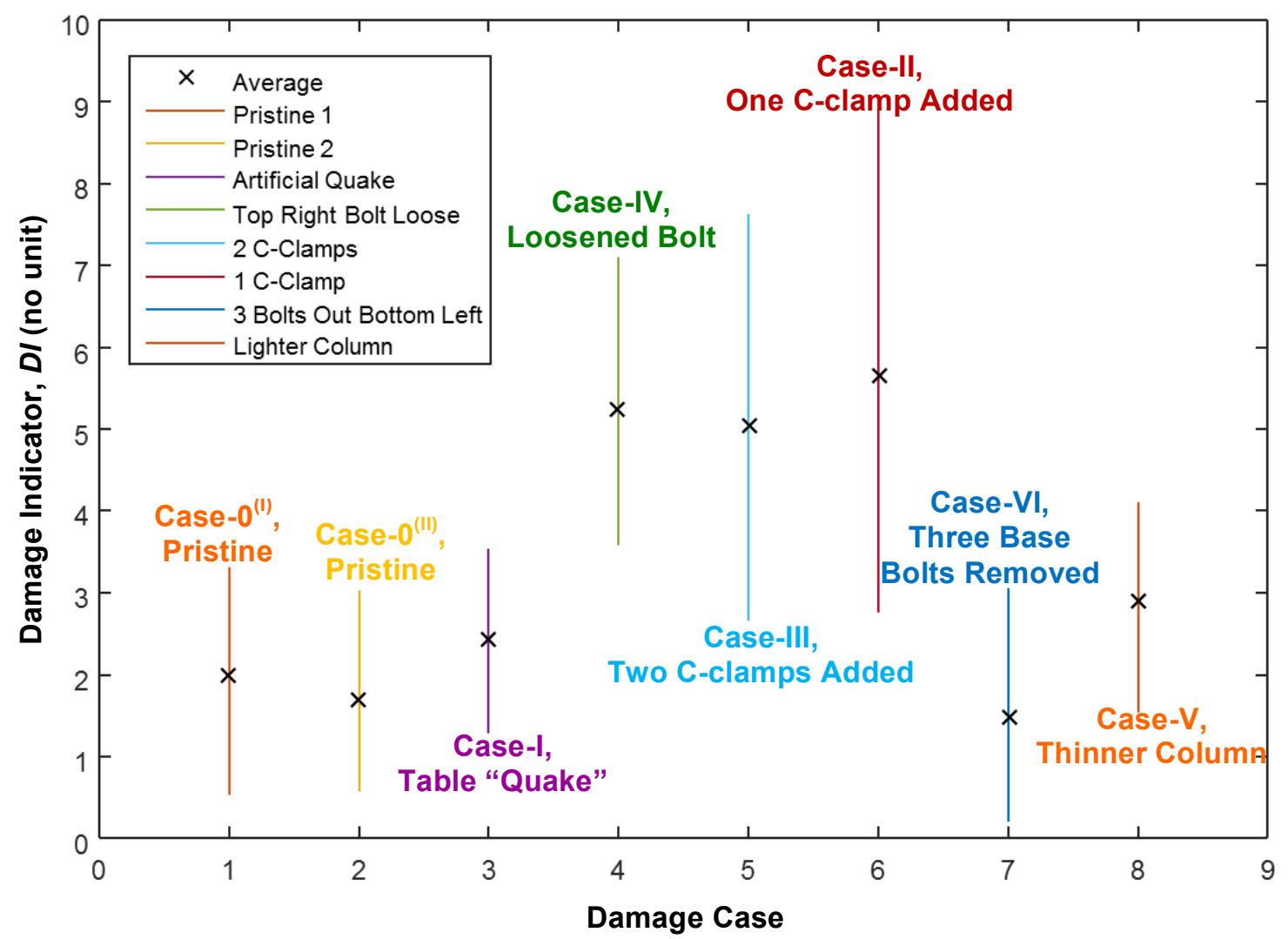

Figure 11. Ranges of damage indicators that account for modeling uncertainty (at $\alpha=1)$. 